\title{
Chasing Hope: When Are Requests for Hospital Transfer a Place for Palliative Care Integration?
}

\author{
Leah B Rosenberg, MD*, Juliet C Jacobsen, MD
}

Division of Palliative Care and Geriatrics, Massachusetts General Hospital, Boston, Massachusetts.

"I don't think she'll ever make it home again."

I stood at the nursing station with two staff nurses from our medical ward. The patient, a woman with metastatic cancer and acute respiratory failure, had just arrived by a medical helicopter from an out-of-state community hospital. At the previous hospital, days had stretched into weeks, and she was not getting better. Limited documentation told us that the patient's family sought further options at our facility. Beyond that, we knew little about the conversations that had transpired. The transfer had been arranged and off she went, arriving on our helipad in worsening respiratory distress, now needing a higher level of supplemental oxygen and teetering on the edge of endotracheal intubation. Above all, she was extremely uncomfortable from shortness of breath, and an urgent palliative care consult was placed a few hours after she touched down.

I arrived at the bedside to meet the patient and family. In these circumstances, I often feel that a palliative care consult is not the miracle that patients seek, but the grim consolation prize behind door two of a tragic and exhausting game show of a hospital transfer. I read the family's dismayed facial expressions as they gazed at my badge reading "Palliative Care" and imagined that they would have preferred a different answer to their question that led them here: "Is this all that can be done?"

The hope of patients and families is a precious thing. As a palliative care physician, I recognize that my role is never to take away hope but rather reframe the wish for recovery into the realm of the achievable. Some people can pivot when the hospital transfer happens. They feel that they have "done all they could" and searched the earth for the reversal of their medical circumstances. I empathize with the wish to try everything and respect the motivation that lies behind it. I also see the cost of chasing hope.

In 2017, 1.5 million patients were transferred, comprising $3.5 \%$ of all hospital admissions. ${ }^{1}$ Although the majority of hospital transfers are medically appropriate and hold the possibility of treatment options unavailable at smaller facilities, there are also significant drawbacks. Transfers have been identified as risky times for adverse events, are often expensive, and are associated with higher mortality and longer length of stay. ${ }^{2}$ There are often lapses in documentation and provider com-

Corresponding Author: Leah B. Rosenberg, MD;

E-mail: Ibrosenberg@partners.org.

Published online first December 18, 2019

Received: June 14, 2019; Revised: October 2, 2019; Accepted: October 7, 2019 (C) 2020 Society of Hospital Medicine DOI 10.12788/jhm.3341 munication, and handoff practices vary widely between hospitals. ${ }^{3}$ Some transferred seriously ill patients become functionally trapped in the accepting hospital. Patients arrive too sick to undergo any meaningful disease-directed therapy and too medically tenuous to return to their home community.

When patients and families seem overly hopeful about what a transfer might provide, the request for transfer may indicate a deeper need for empathic understanding. Clinical conversations about "what can be done" typically focus on medical aspects and often miss a critical element-a complete exploration of a seriously ill patient's prognostic awareness.

In palliative care, we use the term prognostic awareness to define patients' dynamic understanding of their prognosis in terms of likely longevity and quality of life. Accurate prognostic awareness-where there is concordance between a patient's worries and the medical facts as understood by their treatment team-has been associated with enhanced quality of life and mood when patients have enough emotional reserve to actively cope with the illness; for example, by reframing things in a positive light. ${ }^{4,5}$ However, when patients are struggling to cope, talking about the prognosis is hard for patients and for their clinicians, who often accurately perceive their patient's struggle and delay conversations, hoping that more time will help their patient better adapt and prepare to talk about it.6,7 However, delaying conversations makes it even harder for patients and families to develop accurate prognostic awareness, leaving them unprepared when medical decisions arise. ${ }^{8}$ Such delays have a particularly strong impact in nononcology care, where a more unpredictable illness trajectory makes it even harder for patients to understand and prepare for what might happen.

When seriously ill patients and families consider transfer to a tertiary medical center in a situation of medical crisis, it can be a good time to pause. Palliative care specialists are trained to communicate around these difficult points of transition, but generalist clinicians already involved in the patient's care can also sensitively explore patients' prognostic awareness as it relates to the hospital transfer. ${ }^{9}$ In the Table, The phrases mentioned suggest language that is helpful in broaching such discussions, which assesses the patient's illness understanding, hopes, and worries. Asking about patients' hopes for their illness enables clinicians to quickly know some of their most important priorities. Giving patients the permission to be future-oriented and positive also supports them to cope in these challenging conversations. Asking patients to identify two or three hopes places their most optimistic hopes within a larger context and can lead to a discussion of the potential tradeoffs of the transfer. ${ }^{10}$ For example, the hope for a little more time from treatments avail- 
TABLE. How to Assess Prognostic Awareness at the Time of Impending Hospital Transfer

\begin{tabular}{ll}
\hline Three Parts of Prognostic Awareness & Suggested Language \\
\hline Assess illness understanding & What is your understanding of your health or current medical situation? \\
\hline Explore hopes & Can you share more about what you are hoping for with this illness? \\
& Are there other hopes that you have related to this illness? \\
\hline Explore worries & What are your biggest worries? \\
& If no further treatments were possible at the next facility, what would then be your biggest worries? \\
& What would the potential drawbacks of a transfer be from your point of view?
\end{tabular}

able through transfer may be at odds with the hope to spend as much time as possible with family. Once the patient's hopes are better understood, the clinician can then ask about worries. Most seriously ill patients are deeply (often silently) worried about the future, and when asked, can articulate worries about dying that can be the foundation for an honest conversation about the likely course of the hospital transfer.

With empathic assessment, several patients can speak honestly with their clinicians about their illness, including the pros and cons of hospital transfer. However, some continue to struggle, often asking clinicians to remain positive and not endorsing any worry. We describe these patients as having low prognostic awareness. ${ }^{11}$ With such patients, palliative care expertise may be needed to ensure that patients and their families have the information they need to engage in informed medical decision-making. There are emerging models for distance palliative care integration, which may be helpful in these situations. ${ }^{12}$ If these technologies became common in practice, frontline clinicians may increasingly find virtual consultation helpful in working with patients to develop more accurate prognostic awareness. There is also the possibility of clinician-to-clinician electronic consultation, or peer coaching, where the patient is not seen directly by the consultant, but expert advice is offered to the local provider. ${ }^{13}$ Although both these innovations offer service that currently may not be available in certain care settings, in-person consultation remains the gold standard. If a nuanced discussion cannot be had, palliative care expertise may be the reason for transfer.

Back at our hospital, I met with the patient and her partner. There were tradeoffs to be made and hard truths to be acknowledged. In this unfamiliar place, with caregivers she had met just hours before, the patient changed her resuscitation order to allow for a natural death. She passed away later that evening, surrounded by her immediate family but far away from the community that had held her throughout her illness. I reflect on the loss that can come with choosing "everything" when efforts are often better spent on ensuring comfort. Although hospital transfer is often the right answer for seriously ill patients seeking diagnostic and therapeutic options unavailable at their home medical centers, the question should also be an impetus for a nuanced assessment of patients' prognostic awareness to prepare patients if things do not go as hoped or to enlist palliative care expertise for those struggling to cope. As there is a workforce shortage of palliative care providers, particularly in smaller and rural American hospitals, frontline hospitalist clinicians may find themselves increasingly playing a critical role in discussions where transfer is considered. By assessing patients' prognostic awareness through thoughtful, compassionate inquiry in these moments of transition, we can support informed medical decision-making.

Disclosure: The authors do not report any conflicts of interest relevant to this piece.

\section{References}

1. Hernandez-Boussard T, Davies S, McDonald K, Wang NE. Interhospital facility transfers in the United States: a nationwide outcomes study. J Patient Saf. 2017;13(4):187-191. https://doi.org/10.1097/PTS.0000000000000148.

2. Ligtenberg JJ, Arnold LG, Stienstra Y, et al. Quality of inter-hospital transport of critically ill patients: a prospective audit. Crit Care. 2005;9(4):R446-R451. https://doi.org/10.1097/PTS.0000000000000148.

3. Payne CE, Stein JM, Leong T, Dressler DD. Avoiding handover fumbles: a controlled trial of a structured handover tool versus traditional handover methods. BMJ Qual Saf. 2012;21(11):925-932. https://doi.org/10.1136/bmjqs-2011-000308.

4. Nipp RD, Greer JA, El-Jawahri A, et al. Coping and prognostic awareness in patients with advanced cancer. J Clin Onc. 2017;1(22):2551-2557. https://doi. org/10.1200/JCO.2016.71.3404.

5. El-Jawahri A, Traeger L, Park, et al. Associations among prognostic understanding, quality of life, and mood in patients with advanced cancer. Cancer. 2014;120(2):278-285. https://doi.org/10.1002/cncr.28369.

6. Temel JS, Greer JA, Admane S, et al. Longitudinal perceptions of prognosis and goals of therapy in patients with metastatic non-small-cell lung cancer: results of a randomized study of early palliative care. J Clin Oncol. 2011;29(17):2319-2326. https://doi.org/10.1200/JCO.2010.32.4459.

7. Bernacki R, Paladino J, Neville BA, et al. Effect of the serious illness care program in outpatient oncology: a cluster randomized clinical trial. JAMA Intern Med. 2019;2019179(6):751-759. https://doi.org/10.1001/jamainternmed.2019.0077.

8. Jackson VA, Jacobsen J, Greer JA, et al. The cultivation of prognostic awareness through the provision of early palliative care in the ambulatory setting: a communication guide. J Palliat Med. 2013;16(8):894-900. https://doi. org/10.1089/jpm.2012.0547.

9. Lakin JR, Jacobsen J. Softening our approach to discussing prognosis. JAMA Intern Med. 2019;179(1):5-6. https://doi.org/10.1001/jamainternmed.2018.5786.

10. Nipp RD, El-Jawahri A, Fishbein JN, et al. The relationship between coping strategies, quality of life, and mood in patients with incurable cancer. Cancer. 2016;122(13):2110-2116. https://doi.org/10.1002/cncr.30025.

11. El-Jawahri $A$, Traeger $L$, Park ER, et al. Associations among prognostic understanding, quality of life, and mood in patients with advanced cancer. Cancer. 2014;120(2):278-285. https://doi.org/10.1002/cncr.28369.

12. Tasneem S, Kim A, Bagheri A, Lebret J. Telemedicine video visits for patients receiving palliative care: A qualitative study. Am J Hosp Palliat Care. 2019;36(9):789-794. https://doi.org/10.1177/1049909119846843.

13. Lustbader D, Mudra M, Romano $C$, et al. The impact of a home-based palliative care program in an accountable care organization. J Palliat Med. 2017;20:23-28. https://doi.org/10.1089/jpm.2016.0265. 